\title{
SAÚDE BUCAL E CUIDADOS ODONTOLÓGICOS PARA COM O BEBÊ: UMA ANÁLISE SOB A PERSPECTIVA DA GESTANTE ${ }^{1}$
}

\author{
ORAL HEALTH AND DENTAL CARE FOR THE BABY: \\ AN ANALYSIS FROM THE PREGNANT PERSPECTIVE
}

\section{Flaviane Martins Vaz Nunes dos Santos ${ }^{2}$, Waleska Fernanda Souto - Nóbrega ${ }^{3}$, Gustavo Correia Basto da Silva ${ }^{4}$ e Smyrna Luíza Ximenes de Souza ${ }^{5}$}

\section{RESUMO}

Objetivo: Investigar o nível conhecimento das gestantes atendidas nas Unidades Básicas de Saúde da Família do município de Borborema-PB sobre saúde bucal durante a gravidez e sobre os cuidados odontológicos do bebê. Metodologia: Foi realizado um estudo de caráter transversal, através de uma entrevista estruturada composta por 25 questões. Os dados foram tabulados no software Microsoft Excel e em seguida foi realizada estatística descritiva no SPSS 22.0, demonstrada através de frequências absolutas e relativas. Resultados: A amostra foi composta por 46 gestantes, das quais $28,26 \%$ estavam no nono mês de gestação e $78,26 \%$ foram orientadas a ir ao dentista durante a gravidez. Além disso, 23,91\% relataram tanto sentir dor de dente como ter sangramento gengival. Acerca de sua própria higiene bucal, 58,70\% das gestantes escovam os dentes três vezes ao dia e $43,38 \%$ usam fio dental. Da totalidade, $60,87 \%$ sabem o que usar para higienizar a boca do bebê. Conclusão: Tendo em vista que quase metade das gestantes acredita ser normal sentir dor de dente durante a gestação, evidencia-se que ainda há mitos e crenças a serem superados a respeito da assistência odontológica no período gestacional. Para além disso, o conhecimento das gestantes sobre os cuidados odontológicos para com o bebê mostra a pouca orientação recebida durante o período gestacional.

Palavras-chave: Gravidez, Saúde Bucal, Tratamento Odontológico.

\section{ABSTRACT}

Objective: To investigate the level of knowledge of pregnant women attended at Basic Family Health Units in the city of Borborema-PB about oral health during pregnancy and about the baby's dental care. Methodology: A cross-sectional study was carried out, through a structured interview consisting of 25 questions. Data were tabulated in Microsoft Excel software and then descriptive statistics were performed using SPSS 22.0, demonstrated through absolute and relative frequencies. Results: The sample consisted of 46 pregnant women, of which $28.26 \%$ were in the ninth month of pregnancy and $78.26 \%$ were instructed to go to the dentist during pregnancy. In addition, 23.91\% reported both experiencing toothache and gingival bleeding. About their own oral hygiene, $58.70 \%$ of pregnant women brush their teeth three times a day and $43.38 \%$ use dental floss. Of the total, $60.87 \%$ know what to use to clean the baby's mouth. Conclusion: Considering that almost half of pregnant

1 Trabalho de Conclusão de Curso - Graduação em Odontologia.

2 Graduanda em Odontologia, Universidade Estadual da Paraíba - UEPB, Araruna - PB, Brasil. Email: flavianevaz54@, gmail.com

3 Doutoranda em Odontologia, Professora substituta do departamento de Odontologia, Universidade Estadual da Paraíba UEPB, Campina Grande - PB, Brasil. Email: drawaleskasouto@gmail.com

4 Doutorando em Odontologia. Professor substituto do departamento de Odontologia, Universidade Estadual da Paraíba UEPB, Araruna - PB, Brasil. Email: gugacorreiaa@gmail.com

5 Doutoranda em Odontologia. Professora substituta do departamento de Odontologia, Universidade Estadual da Paraíba UEPB, Araruna - PB, Brasil. Email: smyrnaximenes@gmail.com 
women believe that it is normal to feel toothache during pregnancy, it is evident that there are still myths and beliefs to be overcome regarding dental care during pregnancy. In addition, the knowledge of pregnant women about dental care for the baby shows the little guidance received during the gestational period.

Keywords: Pregnancy, Oral Health, Dental Care.

\section{INTRODUÇÃO}

A saúde bucal é parte indissociável da saúde geral do indivíduo, e ambas são consideradas como diretamente dependentes de questões socioculturais e econômicas, considerando aspectos como rede de esgoto, acesso à água de qualidade, conhecimento adquirido, dentre outros (BRASIL, 2002).

No que diz respeito à gestação, deve-se considerar que intensas mudanças fisiológicas e hormonais ocorrem no corpo da mulher neste período. Alterações essas que tem relação direta com manifestações na cavidade oral. Os enjoos matutinos, a ingestão de carboidrato aumentada e o consumo de diversas refeições ao dia associados à mesma quantidade de escovação ou até a redução desta, favorecem o aparecimento de cáries e inflamações gengivais nas gestantes (ZEMOLIN et al., 2017).

Uma das maneiras de evitar o aumento desses índices é investir em educação em saúde, incentivando as famílias a adotar novos hábitos de higiene oral bem como dietéticos, além de ensinar as corretas formas de higienização bucal - escovação, uso de fio dental e enxaguantes bucais (SILVA et al., 2013).

No entanto, questões culturais ainda influenciam negativamente as mulheres grávidas. Muitas gestantes insistem em acreditar que é normal sentir dor de dente durante a gravidez, que nesse período a perda de um dente pode ser algo previsível devido à falta de cálcio que está sendo direcionada ao bebê. Além disso, é possível encontrar profissionais que, devido à insegurança ou à falta de conhecimento especifico, restringem-se a procedimentos de urgência, baseados em técnicas curativas ou medicações paliativas, negligenciado as atividades de prevenção e promoção de saúde para esse público (CORREIA; SILVEIRA, 2011).

Martins et al. (2019), demonstraram em seu estudo que as gestantes ainda demonstram pouco conhecimento quanto à importância da realização do pré-natal odontológico, o que gera consequências não só nesta mas no futuro bebê, sugerindo, destarte, a necessidade de maior atenção materno-infantil na Odontologia, bem como uma maior integração entre classe médica e odontológica, pautando o atendimento na educação em saúde durante este período.

Bastiani, Cota e Provenzano (2010) afirmam que o período gestacional é um momento ideal para as mulheres serem orientadas sobre promoção e prevenção de saúde. Durante a gravidez elas estão aptas a mudanças, a adquirir novos hábitos alimentares, de higiene, de cuidados com o próprio corpo, em busca de oferecer o melhor ao bebê que estão gerando.

Para além disso, Fumagalli et al. (2021) apontam o fato de que, além do nível baixo de conhecimento sobre a influência do período gestacional em sua saúde bucal e na de seu bebê, estas ainda 
acreditam que há contra- indicações no que tange à gestante de procurar o dentista nesse período e de realizar procedimentos odontológicos, tendo em mente que estes podem ser prejudiciais.

Nesse ínterim, torna-se extremamente necessário que toda gestante atendida na rede pública de saúde realize o pré-natal odontológico, no qual os profissionais de saúde bucal devem estar aptos a oferecer um atendimento integral a essas gestantes (HANNA; NOGUEIRA; HONDA, 2010).

Para além disso, é necessário orientar essas mulheres sobre os cuidados com a higiene oral e sua relação com a saúde da criança, sobre as alterações hormonais que ocorrem durante a gravidez e estimular a amamentação explicando os benefícios que ela traz tanto para a mãe quanto para o filho (ZEMOLIN et al., 2017). Porém, a criação de programas preventivos deve pautar-se no conhecimento do nível atual de conhecimento das gestantes sobre a importância do pré-natal odontológico, sendo necessária a realização de pesquisas de cunho investigativo sobre este determinado tema.

Destarte, o objetivo desta pesquisa foi investigar o nível de conhecimento das gestantes atendidas nas UBS do município de Borborema, PB sobre sua própria saúde bucal durante o período gestacional bem como sobre os cuidados odontológicos para com o bebê.

\section{MATERIAL E MÉTODOS}

Este estudo foi de caráter transversal e descritivo. A coleta de dados foi realizada através da realização de uma entrevista estruturada, cujas 25 questões foram elaboradas pelos próprios autores, baseada na literatura de base e nos conhecimentos aprendidos nas disciplinas já cursadas durante a graduação.

Para realização da pesquisa, foi eleito o município de Borborema - PB, localizado na região geográfica imediata de Guarabira, com população estimada de 5.263 habitantes para o ano de 2019 e que conta atualmente com três unidades básicas de saúde, duas urbanas e uma rural8. A amostra deste estudo foi constituída por 46 gestantes compareceram a unidade básica de saúde da família (UBSF) para realização de consulta pré-natal.

$\mathrm{O}$ atendimento a gestantes se dá em todas as UBS do município, tanto pela equipe médica como pela odontológica mediante encaminhamento da enfermeira. Há no município um grupo de gestantes, chamado "Valorizando a Vida", que se reúno no Centro de Referência da Assistência Social (CRAS), havendo interação entre esse grupo e as equipes da UBS para palestras educacionais. No município não há um programa de pré-natal odontológico propriamente dito.

Para a coleta de dados foi realizada uma entrevista estruturada composta por 25 questões que abordavam temas como a saúde bucal da própria gestante, dados gerais de sua gestação, bem como sobre o seu conhecimento acerca da saúde bucal do bebê. Os questionários foram aplicados às gestantes em forma de entrevista nas dependências das UBSF por uma única pesquisadora, individualmente. Estas, foram abordadas no momento em que compareceram para realizar consulta médica/de enfermagem 
de rotina. De forma voluntária, foram conduzidas até uma sala silenciosa, na qual a entrevista pode ocorrer preservando-se sua privacidade.

Posteriormente a coleta de dados, estes foram organizados e armazenados em planilhas do software Microsoft Excel. Em seguida, os dados foram analisados através do software SPSS 22.0, no qual foi realizada estatística descritiva, a qual está demonstrada através de frequências relativas e absolutas, no formato de tabela.

Por se tratar de uma pesquisa com seres humanos, o estudo seguiu as proposições da Declaração de Helsinque. Não obstante, o presente estudo foi registrado na Plataforma Brasil e seguidamente submetido à avaliação pelo Comitê de Ética em Pesquisa da Universidade Estadual da Paraíba, sob o parecer de número 3.296.827 (CAAE: 12805719.8.0000.5187). Todos os participantes foram informados do caráter e objetivo do estudo e participaram voluntariamente através da assinatura de um Termo de Consentimento Livre e Esclarecido - TCLE.

\section{RESULTADOS}

Os resultados obtidos se referem a uma amostra de 46 gestantes entrevistadas que estavam sendo atendidas nas UBSF de Borborema-PB e representaram a totalidade de gestantes do município quando da aplicação do questionário nos meses de julho de agosto de 2019.

O maior percentual de gestantes se encontrava no nono mês de gestação $(28,26 \%)$, sendo que, da totalidade, 56,53\% não estavam em sua primeira gestação. Acerca de orientações recebidas, $78,26 \%$ gestantes afirmaram ter sido orientadas à visitarem o dentista e $60,87 \%$ afirmaram terem recebido informações acerca da saúde bucal (Tabela 1).

Tabela 1 - Gestante e tratamento odontológico, Borborema - PB, 2019.

\begin{tabular}{lcc}
\hline Variáveis & N & \% \\
\hline Período gestacional & & \\
Primeiro mês & 2 & 4,35 \\
Segundo mês & 5 & 10,87 \\
Terceiro mês & 5 & 10,87 \\
Quarto mês & 6 & 13,04 \\
Quinto mês & 3 & 6,52 \\
Sexto mês & 4 & 8,70 \\
Sétimo mês & 8 & 17,40 \\
Oitavo mês & 13 & 28,26 \\
Nono mês & & \\
\hline Primeira gestação & 20 & 43,38 \\
\hline Sim & 26 & 56,52 \\
Não & & \\
\hline Tinha hábito de ir ao dentista antes da gestação? & 25 & 54,35 \\
\hline Sim & 21 & 45,65 \\
\hline Não
\end{tabular}




\begin{tabular}{lcc}
\hline Foi orientada a ir ao dentista durante a gravidez? & & \\
\hline Sim & 36 & 78,26 \\
Não & 10 & 21,74 \\
\hline Acha que gestantes podem fazer tratamento odontológico? & 37 & 80,43 \\
\hline Sim & 9 & 19,57 \\
Não & & \\
\hline Recebeu orientação sobre saúde bucal durante a gravidez? & 28 & 60,87 \\
\hline Sim & 18 & 39,13 \\
\hline Não
\end{tabular}

Fonte: Construção do Autor, 2019.

Em se tratando da gestante e sua própria saúde bucal, a tabela 2 nos informa que 30,43\% perceberam alguma alteração na boca durante a gestação, sendo a dor de dente e o sangramento gengival presentes em 23,91\% da amostra. Quanto aos hábitos de escovação, a maioria relatou escovar os dentes três vezes ao dia $(58,70 \%)$, porém menos da metade usam fio dental $(43,38 \%)$.

Tabela 2 - Gestante e saúde bucal, Borborema - PB, 2019.

\begin{tabular}{|c|c|c|}
\hline Variáveis & $\mathbf{N}$ & $\%$ \\
\hline \multicolumn{3}{|c|}{ Notou alguma alteração na boca durante a gravidez? } \\
\hline Sim & 14 & 30,43 \\
\hline Não & 32 & 69,57 \\
\hline \multicolumn{3}{|c|}{ Você acha normal sentir dor de dente na gravidez? } \\
\hline Sim & 22 & 47,83 \\
\hline Não & 24 & 52,17 \\
\hline \multicolumn{3}{|c|}{ Você sente dor de dente? } \\
\hline Sim & 11 & 23,91 \\
\hline Não & 35 & 76,09 \\
\hline \multicolumn{3}{|c|}{ Sua gengiva sangra ao escovar os dentes? } \\
\hline Sim & 11 & 23,91 \\
\hline Não & 35 & 76,09 \\
\hline \multicolumn{3}{|c|}{ Você sente mobilidade em algum dente? } \\
\hline Sim & 4 & 8,70 \\
\hline Não & 42 & 91,30 \\
\hline \multicolumn{3}{|c|}{ Quantas vezes você escova os dentes ao dia? } \\
\hline 1 & 6 & 13,04 \\
\hline 2 & 10 & 21,74 \\
\hline 3 & 27 & 58,70 \\
\hline 4 & 3 & 6,52 \\
\hline \multicolumn{3}{|c|}{ Você usa fio dental? } \\
\hline Sim & 20 & 43,38 \\
\hline Não & 26 & 56,52 \\
\hline
\end{tabular}

Fonte: Construção do Autor, 2019.

A tabela 3 traz informações acerca do que a gestante sabe sobre os cuidados bucais para com seu bebê. Em números percentuais, $69,57 \%$ das gestantes relacionaram tanto sua alimentação quanto sua saúde bucal como tendo influência na saúde do bebê. Sobre o uso da mamadeira, 52,17\% pretendem dar aos seus bebês, enquanto $58,70 \%$ pretender fazer uso da chupeta. 
Algumas perguntas presentes na tabela 3 foram desdobradas caso a resposta fosse "sim", na possibilidade de uma posterior resposta subjetiva. Em se tratando dos cuidados com a boca do bebê, $73,91 \%$ das mães disseram saber o que fazer, e destas a maioria $(19,57 \%)$ citou o ato de escovar os dentes, seguido do ato de limpar a língua (13,04\%) e limpar após cada mamada (10,87\%).

Pouco mais da metade (54,35\%) relatou que sabe quando deve começar a higienizar a boca do bebê, e dentre elas 23,91\% disseram "assim que amamentar", seguido de outras respostas como "assim que nascer" (10,87\%), "quando nascer o primeiro dente" (6,52\%) e "após um ano de idade" (4,35\%).

Responderam que sabem o que usar para higienizar a boca do bebê 54,35\%, e dentre estes, a maioria (41,30\%) disse ser com fralda molhada com água. Também, 4,35\% disseram que usariam mel rosado com cotonete para esta higienização, e esta mesma quantidade de mães usaria escova.

Tabela 3 - Cuidados da gestante para com o bebê, Borborema - PB, 2021.

\begin{tabular}{llc}
\hline Variáveis & N & \% \\
\hline Sua alimentação pode influenciar na saúde do seu bebê? & 32 & 69,57 \\
Sim & 14 & 30,43 \\
Não & 32 & \\
\hline Você pretende dar chupeta ao seu bebê? & 14 & 39,57 \\
\hline Sim & & \\
Não & 27 & 58,70 \\
\hline Você sabe que cuidados deve ter com a boca de seu bebê? & 19 & 41,30 \\
\hline Sim & 25 & 54,35 \\
Não & 21 & 45,65 \\
\hline Você pretende dar mamadeira ao seu bebê? & & \\
\hline Sim & 28 & 60,87 \\
Não & 18 & 39,13 \\
\hline Você sabe quando deve começar a higienizar a boca de seu bebê? & & \\
\hline Sim & 19 & 41,30 \\
Não & 27 & 58,70 \\
\hline Você sabe com qual idade a criança pode ir ao dentista? & & \\
\hline Sim & & \\
Não & & \\
\hline
\end{tabular}

Fonte: Construção do Autor, 2019.

\section{DISCUSSÃO}

Sabe-se que o pré-natal odontológico é ainda algo recente na Odontologia, no entanto, este possui uma gama de detalhes que não podem ser deixados de lado pelo cirurgião-dentista. Martins et al. (2013), em sua pesquisa, observaram que 12,3\% dos dentistas pesquisados não tinham conhecimento sobre ele, apesar de atenderem gestantes. Bastiani, Cota e Provenzado (2010) denotaram que apenas $21 \%$ dos dentistas no estado do Paraná faziam parte de programas de pré-natal.

Da totalidade de 46 gestantes que participaram desta pesquisa, o maior percentual, de 28,26\%, se encontrava no nono mês gestacional, e apenas 4,35\% estavam no segundo mês gestacional. 
Olivo (2013) discorre acerca da crença do não atendimento odontológico de gestantes, e pontua que ainda há dentistas que acabam por não atender gestantes em sua rotina de atendimentos, somente as atendem em situações emergenciais.

A American Dental Association (ADA, 2008), ainda em 2008, já preceituava que todos os dentistas deveriam saber informar a suas pacientes acerca da segurança na realização de atendimentos odontológicos, e que todos os profissionais de saúde deveriam orientar os pacientes acerca da saúde bucal e da saúde bucal de seus bebês.

Martins et al. (2013) pontuam que a hesitação dos dentistas em tratar gestantes pode ser atribuída a alguns fatores, dentre eles a lacuna acerca desse assunto na sua formação acadêmica aliado a um contexto de crenças populares por parte das próprias gestantes, o que cria um clima de insegurança. Corroboram este pensamento Vasconcelos et al. (2012), complementando que o atendimento odontológico a gestantes chega a ser um assunto controverso, especialmente em função dos mitos baseados em crenças antigas sem fundamentação científica, tanto por parte das gestantes como por parte dos dentistas.

Santos-Pinto et al. (2001) constataram em sua pesquisa que apenas 33\% das gestantes receberam orientações acerca de como manter sua saúde bucal, na de Martins e Martins (2002), apenas $37,5 \%$, e na de Cruz et al. (2004), 32,5\%, contrariando os nossos achados, nos quais $60,87 \%$ das gestantes disseram ter recebido orientações sobre saúde bucal. Isto sugere que políticas públicas voltadas para gestantes tem tido resultados com o passar dos anos.

Os resultados demonstraram que 43,38\% das gestantes estavam em sua primeira gestação, números próximos dos encontrados por Santos-Pinto et al.13 - 48,10\% e por Bastiani, Cota e Provenzano (2010) - 48,75\%. Esta característica é importante, pois mostra que as mamães de "primeira viagem" estão interessadas em receber informações acerca de sua gestação bem como do bebê.

Outro dado que denota bons resultados das políticas públicas implementadas, é que 80,43\% das gestantes acharam que podem fazer tratamento odontológico, número bem acima dos encontrados por Bastiani, Cota e Provenzano (2010), que foi de 68,75\%.

A gestação em si não é responsável pelo surgimento de alterações na cavidade bucal. Porém os frequentes enjoos e consequentes vômitos, que são comuns especialmente no primeiro trimestre da gestação, podem gerar modificação no equilíbrio do $\mathrm{pH}$ bucal e levar à exacerbação de processos cariosos e afecções gengivais. Saliente-se também a questão hormonal da gestante, em especial os altos níveis de progesterona, que aumentam a permeabilidade dos vasos sanguíneos, fato que acaba por deixar a região gengival mais propícia aos microrganismos patogênicos presentes no biofilme (MARTINS et al., 2002).

Além dos fatores supracitados, a falta de conhecimento da mãe sobre saúde bucal e formas de prevenção dos agravos podem acarretar em diversas patologias bucais nos bebês, como a cárie de mamadeira, tornando imprescindível a inclusão precoce dos cuidados com a saúde bucal instruída por 
um profissional ou por intermédio de programas educativos, a fim de prevenir tais malefícios futuros (VIEIRA; SILVA, 2019).

Bastiani, Cota e Provenzano (2010) encontraram em sua pesquisa que 48,75\% das gestantes achavam que é normal ter cárie durante a gravidez, e acreditavam que a manifestação de lesões cariosas se dava ao motivo de os dentes ficarem mais fracos devido à transmissão de cálcio da mãe pra 0 bebê. Nossa pesquisa mostrou que 47,83\% das gestantes acha normal sentir dor de dente na gravidez, confirmando a questão das crenças populares sobre tratamento odontológico em gestantes, inclusive o famoso ditado "a cada gravidez, perde-se um dente"16.

Os nossos achados mostraram que 30,43\% das gestantes perceberam alguma alteração na cavidade bucal durante a gestação. Das alterações percebidas, a dor de dente e o sangramento gengival estiveram presentes em $23,91 \%$ das gestantes - o mesmo percentual para ambos. Granville-Garcia et al. (2007) encontraram valor muito próximo para sangramento gengival - 24,2\%, porém Maia et al. (2007), em sua pesquisa com gestantes no estado do Amazonas, encontraram 41,9\% das gestantes com sangramento gengival.

O hábito da escovação correta e o uso do fio dental devem ser algo com forte presença nas orientações à gestante, especialmente por se saber que muitas vezes há mudanças na dieta alimentar neste momento e pela possível presença de enjoos e vômitos. No que se refere aos hábitos de escovação, 58,70\% das gestantes disseram escovar os dentes três vezes ao dia no nosso estudo e 43,38\% relataram fazer uso do tio dental. Lopes et al. (2016) encontraram um número mais elevado para ambos, de 74,6\% de gestantes que escovavam os dentes três vezes ao dia e de $47 \%$ que faziam uso de fio dental. Já no estudo de Barbieri et al. (2011), o dado encontrado para uso do fio dental foi de 41,5\%. Ao contrário destes números, o estudo de Cruz et al. (2004) encontrou apenas 30,5\% das gestantes escovando os dentes três vezes ao dia.

A última seção do nosso questionário buscou informações acerca da percepção e conhecimento das gestantes para com o bebê, tanto de como sua própria saúde pode influenciá-lo bem como os cuidados em saúde bucal a ter com o bebê. Da totalidade da amostra, 69,57\% disseram que tanto sua alimentação quanto sua saúde bucal podem influenciar a saúde do bebê. Bastiani, Cota e Provenzano (2010) viram que apenas $26,25 \%$ das gestantes de sua pesquisa acreditavam que alterações na cavidade bucal poderiam influenciar a saúde geral do bebê. Lopes et al. (2016) encontraram que 39,7 \% conhecem a associação entre saúde bucal e gravidez. Mais uma vez observamos um caminhar positivo das políticas públicas implementadas no atendimento às gestantes com o passar dos anos.

As respostas acerca dos cuidados com a boca do bebê nos mostraram certa contradição, visto que $73,91 \%$ das gestantes disseram saber que cuidados devem ter com a boca do bebê, porém 60,87\% afirmaram saber o que usar para higieniza-la. Este é um assunto de importância a ser abordado no pré-natal odontológico. Palestras de educação em saúde bucal que tragam ensinamentos sobre os cuidados odontológicos para com o bebê são de grande valia para as gestantes. 
Apesar de, ao longo dos anos, as ações programáticas em saúde bucal para gestantes terem crescido e se estabelecido ao longo do SUS, ainda há muito o que avançar, especialmente no que diz respeito aos cuidados para com os bebês - observamos que pouco mais da metade das gestantes (54,35\%) sabe quando a higienização da boca do bebê deve ter início. É preocupante observar que algumas gestantes ainda pensam que tal higienização deve iniciar apenas após um ano de idade (4,35\%).

A gestação é um estado único e precioso no ciclo de vida da mulher. As gestantes fazem parte, por um período de tempo, dos pacientes especiais na Odontologia por suas mudanças físicas, hormonais e psicológicas que podem vir a criar condições adversas no sistema estomatognático. Por este motivo devem ser consideradas como um grupo com prioridade para as políticas públicas.

\section{CONCLUSÃO}

Pode-se concluir que as gestantes necessitam de mais informações acerca de sua saúde bucal bem como orientações acerca de visitas ao dentista durante o período gestacional. Ainda há mitos e crenças na gestação ligados à Odontologia de uma forma geral, pois encontramos que quase a metade das gestantes acredita ser normal sentir dor de dente neste período. O conhecimento das gestantes sobre os cuidados odontológicos para com o bebê mostra a pouca orientação recebida durante o período gestacional. Ressalta-se a importância da presença do dentista na equipe de profissionais que realizam o pré-natal com gestantes.

\section{REFERÊNCIAS}

AMERICAN DENTAL ASSOCIATION. ADA. Position Statement on Early Childhood Caries, 2008.

BARBIERI W, PERES SV, PEREIRA CB, PERES NETO J, SOUSA ML, CORTELLAZZI KL. Fatores sociodemográficos associados ao grau de conhecimento em saúde bucal de gestantes. Einstein, v. 16, n. 1, p. 1-8, 2018.

BASTIANI C, COTA ALD, PROVENZANO MGA. Conhecimento das gestantes sobre alterações bucais e tratamento odontológico durante a gravidez. Revista Odontologia Clínico-Científica, v. 9, n. 2, p. 155-60, 2010.

BENEDITO FCS, et al. Saúde bucal: conhecimento e importância para a gestante. Revista de Atenção à Saúde, São Caetano do Sul, v. 15, n. 52, p. 43-48, abr./jun. , 2017.

BRASIL. Ministério Da Saúde. As cartas da promoção da saúde. Brasília: Ministério da Saúde, 2002. 
BRASIL. Ministério da Saúde. Coordenação de Saúde Bucal. $8^{\text {a }}$ Conferência Nacional de Saúde. I ${ }^{\text {a }}$ Conferência Nacional de Saúde Bucal. Relatório Final. Brasília: Ministério da Saúde, 1986.

CODATO, L.A.B.; NAKAMA, L.; MELCHIOR, R. Percepções de gestantes sobre atenção odontológica durante a gravidez. Ciência e Saúde Coletiva. v. 13, n. 3, p. 1075-1080, 2008.

CORREIA MB, SILVEIRA JLGC. Percepção da Relação Saúde Bucal e Parto Prematuro entre Membros da Equipe de ESF e Gestantes. Pesquisa Brasileira em Odontopediatria e Clínica Integrada, João Pessoa, v. 11, n. 3, p. 347-55, jul./set. 2011.

CRUZ AAG, GADELHA CGF, CAVALCANTI AL, MEDEIROS AL. Percepção materna sobre a higiene bucal de bebês: Um estudo no hospital Alcides Carneiro, Campina Grande-PB. Pesquisa Brasileira em Odontopediatria e Clínica Integrada. v. 4, n. 3, setembro-dezembro, p. 185-189, 2004.

GRANVILLE-GARCIA AF, LEITE AF, SMITH LEA, CAMPOS RVS. MENEZES VA. Conhecimento de gestantes sobre saúde bucal no município de Caruaru - PE. Revista de Odontologia da UNESP, v. 36, n. 3, p. 243-249, 2007.

HANNA LM, NOGUEIRA AJ, HONDA VY. Percepção das gestantes sobre a atenção odontológica precoce nos bebês. Revista Gaúcha de Odontologia, v. 55, n. 3, p. 271-4, 2007.

IBGE. Cidades: IBGE. Disponível em: https://bit.ly/3mh3qjC. Acesso em: 06, outubro, 2019.

LOPES FF, RIBEIRO TV, FERNANDES DB, CALIXTO RV, ALVES CMC, PEREIRA ALA, PEREIRA AFV. Conhecimento e práticas de saúde bucal de gestantes usuárias dos serviços de saúde em São Luis, Maranhão. Epidemiologia e Serviços de Saúde. Brasília, v. 25, n. 4, p. 819-826, out-dez, 2016.

MAIA AS, SILVA PCS, ALMEIDA MEC, COSTA AMM. Percepção de gestantes do Amazonas em relação à saúde bucal. ConScientiae Saúde. v. 6, n. 2, p. 377-83, 2007.

MARTINS LO, PINHEIRO RPS, ARANTES DC, NASCIMENTO LS, SANTOS JÚNIOR LS. Assistência odontológica à gestante: percepção do cirurgião dentista. Revista Pan-Amazônica de Saúde. v. 4, n. 4, p. 11-18, 2013.

MARTINS RFO, MARTINS ZIO. O que as gestantes sabem sobre cárie: uma avaliação dos conhecimentos de primigestas e multigestas quanto à própria saúde bucal. ABO Nacional, v. 10, p. 278-284, 2002 
MARTINS WLL, et al. Conhecimento de gestantes sobre sua saúde bucal e a do bebê. Revista UNINGÁ, Maringá, v. 56, n. 2, p. 22-33, abr.jun. 2019.

OLIVEO CM. Atendimento odontológico a gestantes: Mitos e Preconceitos por parte dos Cirurgiões Dentistas. Trabalho de Conclusão de Curso (Graduação), Florianópolis, 2013.

SANTOS-PINTO L, UEMA APA, GALASSI IMAS, CIUFF NJO. O que as gestantes conhecem sobre Saúde Bucal? Jornal Brasileiro de Odontopediatria e Odontologia do Bebê, v. 4, p. 429-434, 2001.

SILVA RA, NÓIA NB, GONÇALVES LM, PINHO, JR. CRUZ MC. Avaliação da participação de mães em um programa de prevenção e controle de cáries e doenças periodontais para lactentes. Revista Paulista de Pediatria. v. 31, n. 1, p. 83-89, 2013.

VASCONCELOS RG, VASCONCELOS MG, MAFRA RP, ALVES JÚNIOR LC, QUEIROZ LMG, BARBOSA CAG. Atendimento odontológico a pacientes gestantes: como proceder com segurança. Revista Brasileira de Odontologia. Rio de Janeiro, v. 69, n. 1, p. 120-1244, jan. /jun. 2012.

VIEIRA GFS, SILVA MA. Percepção sobre cuidados bucais dos bebês pelas puérperas no Hospital Universitário Mario Palmério (MPHU). [Trabalho de Coclusão de Curso]. Universidade de Uberaba. Uberaba - MG.

ZEMOLIN AB, SASSI ET, WILLMS GP, RECH JP, DALSOCHIO L. Gestantes: da saúde bucal a saúde geral. Revista Saúde Integrada, v. 10, n. 20, 2017. 